\title{
La estructura de las redes sociales en las compañías del IBEX 35
}

The structure of social networks in IBEX 35 companies

A estrutura das redes sociais nas empresas do IBEX 35
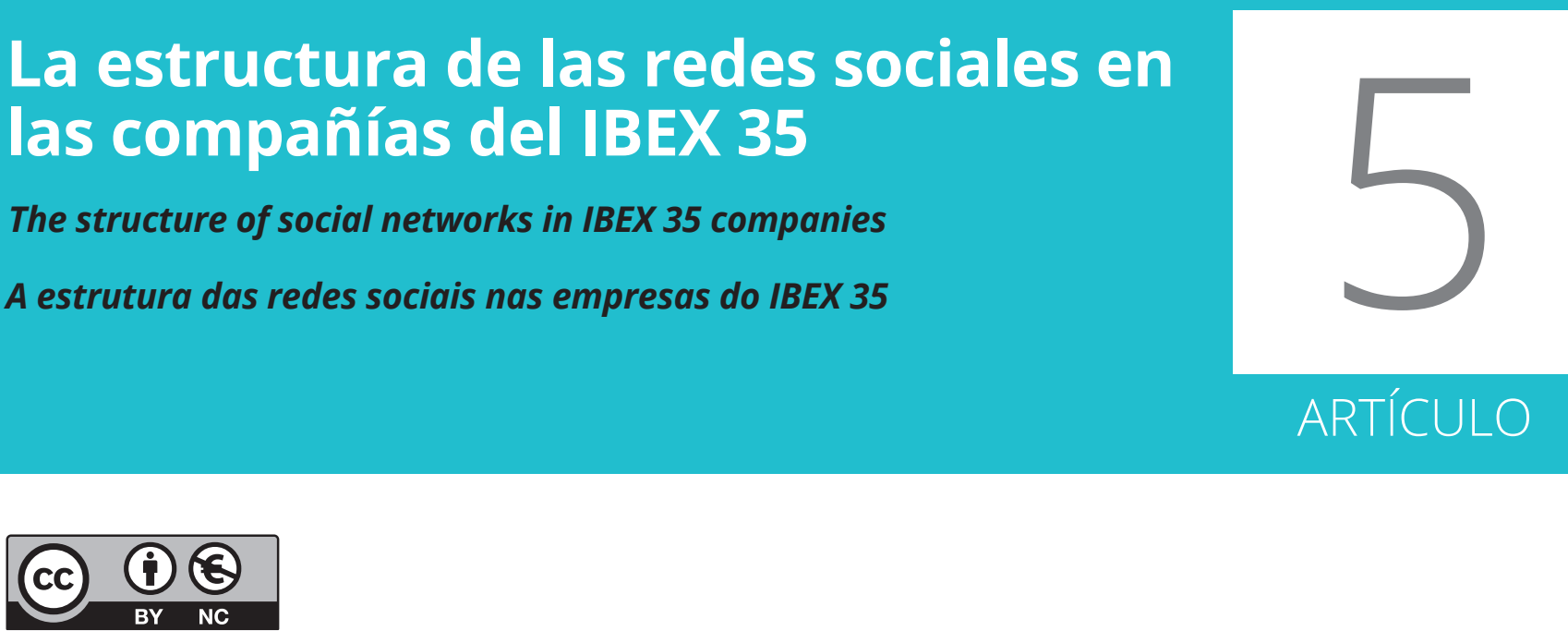

\section{Francisco Leslie López del Castillo Wilderbeek}

Universidad Pompeu Fabra (España)

Máster en Sociedad de la Información por la Universitat Oberta de Catalunya y Doctor en Comunicación por la Universitat Pompeu Fabra. Miembro del equipo de investigación MEDIUM en la misma institución. Documentation Expert en la empresa REBOLD, especializada en el análisis de la comunicación mediante datos. Su producción académica está centrada en el análisis de las acciones de comunicación con especial interés metodológico en el uso de la visualización de datos.

franciscoleslie@alumni.upf.edu

orcid.org/0000-0002-6664-7849

RECEBIDO: 04 de diciembre de 2020 / ACEITO: 29 de enero de 2021

\section{Resumen}

Esta investigación ha elaborado un mapa completo sobre la estructura de redes sociales aceptadas por las empresas del índice IBEX 35. Desde una perspectiva exploratoria se ha indagado la forma en la que estas empresas incluyen las redes sociales en su política de comunicación, desde la indicación en las páginas web oficiales hasta la posible correlación entre tamaño económico y selección de canales. La principal conclusión indica que la función de marketing es predominante para estas empresas y que el tamaño económico tiene una correlación positiva con la selección de redes sociales más informativas en detrimento de las más conversacionales.

\section{PALABRAS CLAVE}

Redes sociales, Blogs, Ibex 35, Interactividad, Conversación, Capitalización.

\section{Abstract}

This research has developed a complete map of the structure of social networks accepted by the companies in the IBEX 35 index. From an exploratory perspective, the way in which these companies include social networks in their communication policy was investigated from their presentation on official web pages 
to the possible correlation between economic size and channel selection. The main conclusion indicates that the marketing function is predominant for these companies and that the economic size has a positive correlation with the selection of more informative social networks to the detriment of the most conversational ones.

\section{KEYWORDS}

Social networks, Blogs, Ibex 35, Interactivity, Conversation, Capitalization.

\section{Resumo}

Esta pesquisa produziu um mapa completo da estrutura das redes sociais aceitas pelas empresas do índice IBEX 35. Numa perspec- tiva exploratória, investigou-se a forma como essas empresas incluem as redes sociais em sua política de comunicação, desde a indicação em páginas oficiais da web até a possível correlação entre tamanho econômico e seleção de canais. A principal conclusão indica que a função de marketing é predominante para essas empresas e que o porte econômico tem correlação positiva com a seleção de redes sociais mais informativas em detrimento das mais conversacionais.

\section{PALAVRAS-CHAVE}

Redes sociais, Blogs, Ibex 35, Interatividade, Conversação, Capitalização.

\section{INTRODUCCIÓN}

En la actualidad, y gracias a la tecnología, la función de la comunicación es estratégica en el sentido en que es capaz de aportar valor al proyecto empresarial de cada organización (Castelló Martínez, 2013). Como indican Klewes et al. (2017) una tendencia relacionada con la transformación digital es el aumento de la conectividad a escala mundial. Este fenómeno supone un reto para la gestión de la comunicación estratégica de las organizaciones ya que obliga a reorientar la relación con los públicos objetivos tanto externos como internos reconociendo la existencia de canales en los que ambos se encuentran en situación de igualdad (Túñez López \& Altamirano, 2015), tanto en las organizaciones privadas como en los organismos públicos. El reciente trabajo de Alonso y López (2019), por ejemplo, observó que para los ayuntamientos, pese a los recursos potencialmente limitados, los medios sociales se sitúan en la zona alta de las opciones escogidas para comunicarse con sus públicos aportando una dimensión bidireccional a la gestión de la comunicación. Por este motivo la formación del director de comunicación debe tener muy en cuenta el nuevo escenario tecnológico, incluyendo la gestión de los «medios digitales, portales corporativos y las redes sociales» (Costa, 2016, p.174).

El paradigma dominante hasta el advenimiento del escenario 2.0 se sustentaba en los canales de comunicación unidireccionales con los que las organizaciones trasladaban información a sus grupos de interés (Macnamara, 2010). Tal y como argumentan Miller y Skinner (2015) la aparición de las redes sociales ha generado una importante pérdida de control sobre las zonas de influencia informativa de las organizaciones, especialmente si se compara con herramientas tradicionales como las notas de prensa o las declaraciones institucionales.

La exhaustiva revisión bibliográfica de Lei et al. (2019) concluyó que las organizaciones usan de forma estratégica las redes sociales con dos fines principales, por un lado, la difusión de in- 
formación relevante y por otro la generación de conversaciones con sus públicos (Okazaki et al., 2020; Wang y Yang, 2020). Ambas actividades se encuentran enmarcadas en la comunicación estratégica de las compañías por su sentido intencional, enfocado a objetivos y planificado por profesionales (Hamid, 2020). Como reflexiona Postman (2009, p.14): «un aspecto clave del éxito en el uso corporativo de las redes sociales es vincular las iniciativas de comunicación a la estrategia de negocio». La adecuada armonización entre ambos elementos podría representar una correlación determinante entre objetivos comunicativos y objetivos corporativos según la literatura previa (Enke \& Borchers, 2019; Kick, 2015). Sin embargo, recientes trabajos empíricos han detectado que en el panorama empresarial español la vertiente informativa sigue siendo hegemónica en comparación con la posibilidad de generar conversaciones con los públicos objetivo (Aced Toledano, 2017; Durántez-Stolle, 2017; Paliwoda-Matiolanska et al., 2020).

En todo caso las potencialidades que ofrecen las redes sociales y el escenario 2.0 en general son extensas para que las organizaciones logren con éxito sus objetivos comunicativos tanto en el ámbito de las relaciones públicas (Allagui \& Breslow, 2016) como en el ámbito de la publicidad (Alalwan, 2018). Por otro lado, la dimensión corporativa de la comunicación en redes sociales es de vital importancia ya que existe constatación empírica según la cual los mensajes en estas plataformas pueden afectar a la percepción que tienen los inversores sobre las organizaciones (Bartov et al., 2018; Cade, 2018; Chen et al., 2014; Tom et al., 2018; Yang \& Yang, 2017).

El modelo desarrollado por Aichner y Jacob (2015) para evaluar el aprovechamiento de las redes sociales por parte de las organizaciones es un ejemplo del interés que han cosechado estos nuevos canales en su vertiente corporativa. Su trabajo se orientó hacia la evaluación del nivel de actividad corporativa en las diferentes plataformas sociales disponibles. Una aportación relevante de su propuesta se basa en la categorización de las diferentes plataformas según su afectación al negocio de las compañías. Concretamente su trabajo segmentó el escenario 2.0 en 13 tipos de plataformas y aplicó un grado de afectación de cada una según la función corporativa implicada. Numerosos trabajos han reconocido esta categorización (Barris, 2019; Korzynski et al., 2020; Poppel, 2020; Uttrup, 2017; Visser \& Sikkenga, 2017). Por ejemplo el estudio de Flores Vizcarra (2019) analizó la generación de contenidos en redes sociales por parte de cuatro empresas de telecomunicaciones de Perú.

Esta investigación se ha propuesto dos objetivos principales. En primer lugar la elaboración de un mapa completo sobre los canales de comunicación digital que forman parte de la estrategia de las empresas del IBEX $35^{1}$ (índice bursátil que mide el comportamiento conjunto de las 35 empresas de la Bolsa española con mayor capitalización, liquidez y volumen negociado). Como canales de comunicación digital se han considerado el uso de redes sociales y de blogs pese a que estos poseen una naturaleza eminentemente más informativa e unidireccional. La inclusión de blogs en igualdad de condiciones que las redes sociales se debe a que se ha verificado empíricamente su consideración semejante por parte de las empresas analizadas y su desvinculación respecto la in-

1 Ordenados por capitalización de mayor a menor: Inditex, Iberdrola, Banco Santander, Amadeus, Telefónica, BBVA, AENA, ENDESA, Naturgy, Ferrovial, REPSOL, Cellnex, Caixabank, IAG, Arcelor, Grifols, ACS, Siemens Gamesa, Red Eléctrica, Mapfre, ENAGAS, Colonial, Merlin, Acciona, Bankinter, Banco Sabadell, Bankia, CIE, Acerinox, Más Móvil, Viscofan, Indra, Melia, Mediaset, Ence (Recuperado el 22 de enero de 2020 de: https://bit.ly/2M5GKmA) 
formación ofrecida en las páginas web oficiales. Tomando como base esta observación se ha convertido la estructura de comunicación digital del conjunto de organizaciones del IBEX en términos de funciones corporativas implicadas aplicando la propuesta de Aichner y Jacob (2015) en forma de coeficientes.

El segundo objetivo establecido ha tenido como propósito la extracción de hallazgos relevantes referidos a las relaciones existentes entre inclusión de redes sociales en la estrategia de comunicación y otros datos de las compañías. Para ello se ha aplicado el cruce de variables externas como el sector en que opera, el tamaño de las compañías y el número de visitas en su web corporativa.

Pese a que este estudio ha empleado un enfoque eminentemente exploratorio, pueden plantearse en origen algunas cuestiones relevantes, como si las compañías del IBEX consideran las redes sociales como canales unidireccionales o conversacionales para comunicarse con sus públicos o si existe correlación entre el tamaño económico de una compañía y el uso que esta hace de las redes sociales como canal de comunicación corporativo.

\section{METOdOLOGÍA}

Este trabajo se ha basado en el análisis de contenido para la observación de la presencia de las empresas del IBEX 35 en las diferentes plataformas sociales disponibles. La extracción de datos se realizó tomando variables cualitativas dicotómicas (presencia / no presencia) y variables cualitativas nominales (presencia, pero sin mención en web corporativa / presencia, pero no con perspectiva corporativa). Estos datos se cuantificaron en hojas de cálculo tanto para la correlación entre ellas como para la conversión en funciones corporativas según la propuesta de Aichner y Jacob (2015). Esta conversión se realizó ponderando las ratios aportadas por estos dos autores según el valor que tiene para las organizaciones el uso de una determinada red social. Concretamente, la valoración se realizó multiplicando el uso de cada red social según si no tenía importancia (0), poca $(0,25)$, media $(0,5)$, alta $(0,75)$ o muy alta (1) en cada una de las funciones corporativas que estos autores delimitaron: I+D, Marketing, Atención al cliente, Ventas, Recursos humanos y Organización.

La detección de los canales de redes sociales incorporados en la estrategia de comunicación corporativa se realizó en primer lugar mediante análisis de contenido de las webs oficiales de cada empresa ${ }^{2}$, y en segundo lugar mediante consulta en las propias plataformas sociales ${ }^{3}$. Desde esta primera consulta se extrajeron datos de los canales que son hegemónicos a nivel cuantitativo: Twitter (número de seguidores), Facebook (número de «me gusta»), Linkedln (número de seguidores) e Instagram (número de seguidores) $)^{4}$.

La consulta de datos sobre el tamaño de cada una de las empresas se realizó accediendo a la web de la Bolsa de Madrid y tomando como referencia la capitalización bursátil (precio de acción por número de acciones en circulación)5.

2 En todos los casos la información y link de acceso se encontraba en la página de inicio excepto en la web de Mediaset en la que era necesario pasar a un segundo nivel de navegación. La consulta se realizó el 22 de enero de 2020.

3 La consulta directa sobre plataformas sociales se realizó una vez cuantificado el total de canales cuantitativamente hegemónicos en las páginas de inicio de las 35 empresas. Se consultaron las plataformas: LinkedIn, YouTube, Instagram, Twitter y Facebook por un lado y se realizó una prospección mediante buscador web de la existencia de blogs oficiales relacionados con cada empresa.

4 Consulta realizada el 22 de enero de 2020.

5 Consulta realizada el 23 de enero de 2020 en https:// bit.ly/2NfTast 
La categorización sectorial de cada empresa se realizó usando como referencia la estructura propuesta por la Bolsa de Madrid 6 . Finalmente la cuantificación del número de visitas a la página web de cada empresa se obtuvo gracias a la plataforma de medición de tráfico web Alexa ${ }^{7}$. Los resultados obtenidos pueden consultarse en detalle en el Anexo 1.

Sobre los resultados finales obtenidos se elaboraron representaciones gráficas y se aplicó visualización de datos. Esta actividad tiene la misión de obtener mediante la observación gráfica de un conjunto de datos una comprensión global de relaciones y de patrones que no son accesibles de otra forma (Torres Ponjuán, 2010).

\section{RESULTADOS}

\subsection{CANALES DE REDES SOCIALES ACEPTADOS POR LAS EMPRESAS DEL IBEX 35}

Como se ha comentado anteriormente, la primera aproximación se realizó con objetivos descriptivos. Mediante análisis de contenido se identificó el total de canales de redes sociales y blogs que forman parte de la estructura de canales de comunicación de cada una de las empresas del IBEX 35. Esta observación, en la que cada empresa está considerada con el mismo valor o peso, convertida en una representación gráfica conduce a la figura 1.

6 1. Petróleo y energía, 2. Mat. Básicos, Industria y Construcción, 3. Bienes de Consumo, 4. Servicios de Consumo, 5. Servicios Financieros, 6. Tecnología y Telecomunicaciones, 7. Servicios inmobiliarios.

7 Consulta realizada el 23 de enero de 2020 en https:// www.alexa.com/siteinfo

\section{Figura 1}

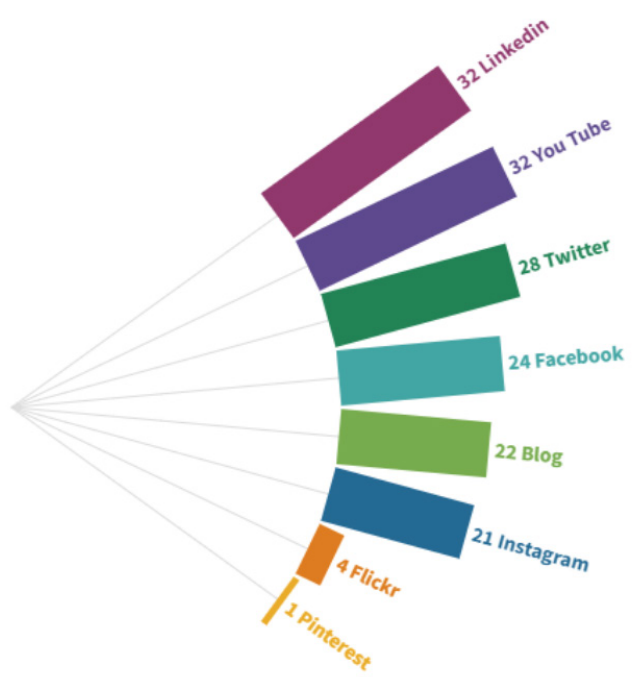

Nota: Gráfica radial organizada cuantitativamente de las redes sociales usadas por parte de las organizaciones del IBEX.

Como puede observarse en la gráfica, si la ponderación de las empresas del IBEX 35 es la misma, el orden de importancia de cada plataforma social es la siguiente: Linkedln y YouTube (empatados), Twitter, Facebook, Blogs, Instagram, Flickr y Pinterest. De esta forma Linkedln se sitúa en la cúspide de los canales de redes sociales por uso corporativo atendiendo al total de empresas del IBEX que lo reconocen como canal propio de comunicación con un total de 32 empresas de las 35 de la muestra. Este resultado desvirtúa por tanto una presunta correlación entre número de usuarios totales de cada plataforma y uso de tipo empresarial o institucional ya que Linkedln se encuentra en la octava posición en la ordenación de redes sociales según el volumen de usuarios activos durante el 2020. Un elemento que refuerza esta desconexión se produce cuando la plataforma Reddit (sitio web de marcadores sociales) que dispone de un mayor número de usuarios no se encuentra reconocido como canal apropiado para estar en las compañías analizadas. Por otro lado, la posición predominante de YouTube, plataforma con mayor uso corporativo de la 
muestra junto con LinkedIn, sí que correspondería parcialmente con el número de usuarios ya que, tomando como variable este dato, la plataforma social de video es la segunda más importante a nivel mundial.

Estos resultados corroboran empíricamente, aunque de manera indirecta, el reciente trabajo de Durántez-Stolle (2017) en el que se concluyó que el componente dialógico de las redes sociales no es el que prima en el uso de las redes sociales de tipo corporativo en comparación con el componente de emisión unidireccional de la información. La sintonía entre ambas argumentaciones se encuentra en que las dos plataformas más usadas por parte del IBEX 35, según los datos de este estudio, fueron YouTube y Linkedln, las que menos ratio de interactividad tienen en comparación con Facebook. La medición basada en actividad diaria coloca a Facebook con una ratio mayor (el 50\% de usuarios lo usan más de una vez al día) en comparación con YouTube (28\%) y especialmente con Linkedln (13\%).

Por otro lado, la cuantificación de canales aceptados por las empresas del IBEX 35 puede ponerse en contexto gracias a un estudio que tomó datos del 2014 en que también observó el número de canales de redes sociales en las compañías del IBEX 35. La tesis doctoral de Aced Toledano (2017) indicó que 17 empresas tenían al menos un blog (respecto a 22 detectados en 2020), 26 tenían página de Facebook (respecto a 24), y 30 tenían perfil de Twitter (respecto a 28).

La comparación en el uso de estos tres canales desde 2014 hasta ahora se encuentra condicionada por el cambio en las compañías que forma el índice bursátil. Sin embargo, desde una perspectiva general puede afirmarse que ha habido una ampliación de los horizontes en la aceptación de las redes sociales por parte de las grandes empresas durante estos seis años. Esta afirmación es debida al aumento en blogs y Facebook por un lado y a que la presunta disminución de canales de Twitter se encuentra potencialmente influida por dos factores. En primer lugar, por una pequeña variación en la metodología aplicada (esta investigación ha observado un uso corporativo o reconocido en la web oficial) y en segundo por el advenimiento de alternativas tecnológicas como el Whatsapp business API que fue lanzado en 2018. Esta plataforma permite una interacción enfocada a la atención al cliente (función no principal que también posee Twitter) y en la actualidad cuenta con una aceptación del 84\% como canal de interacción con los usuarios en medianas y pequeñas empresas según datos de febrero de 2020 aportados por Hootsuite.

Otra cuestión relevante que se observa mediante la descripción de los canales aceptados desde una perspectiva corporativa indica el fenómeno según el cual un número significativo de compañías usan como propias ciertas plataformas, pero no publicitan alguna de ellas en su página web oficial. Este hallazgo es resultado de la aplicación de dos niveles de detección de redes sociales usadas, una primera basada en la indicación de estas en la portada (home page) de cada web y una segunda mediante la búsqueda directa en las plataformas hegemónicas a nivel cuantitativo: Linkedln, YouTube, Instagram, Twitter, Facebook y Blogs.

Concretamente se pudo apreciar que, exceptuando Facebook y Twitter, existe una tendencia proporcionalmente elevada hacia el no reconocimiento en la web corporativa sobre el uso de uno o varios canales de redes sociales. Revisando la actividad de las empresas de la muestra se detectó que el 83\% de las empresas del IBEX 35 omiten en su web oficial el uso de al me nos un canal de redes sociales o blog. Por ejemplo, el $44 \%$ de las empresas del IBEX 
35 que disponen de un canal de YouTube no señalan en su web su existencia, semejantes porcentajes pueden observarse con otros canales como Instagram (38\%) y Linkedln (31\%). Ahora bien, la evidencia empírica más llamativa se produce al analizar los resultados del total de empresas que disponen de un blog corporativo, pero que no lo publicitan directamente en su web oficial. La aplicación de dos niveles de escrutinio señaló que el $77 \%$ de las empresas del IBEX 35 que trasladan contenidos a través de un blog institucional no indicaron este canal en su web oficial. Curiosamente, gran parte de las compañías que sí lo indican claramente pertenecen al sector financiero y usan este canal para ofrecer información sobre tendencias económicas y reportajes sobre responsabilidad social corporativa.

Esta perspectiva mayoritariamente velada queda reforzada cuando los formatos usados en los blogs que no se reconocieron directamente tienden a evitar la presencia promocional de cada una de las entidades que es organizadora y generadora de los contenidos. Por ejemplo, los mismos títulos usados en cada sitio web ofrecen señales sobre una desvinculación respecto al uso meramente corporativo o promocional (por ejemplo Talensostenible.acciona. com en el caso de Acciona o jubilacióndefuturo.es en el caso del BBVA) sin llegar a poseer un sentido fraudulento (flog). Por tanto, puede afirmarse que existe una corriente generalizada hacia la no revelación explícita de los blogs corporativos como canales institucionales en comparación con espacios más interactivos como las redes sociales.

\subsection{CONVERSIÓN DE LAS REDES SOCIALES EN FUNCIONES CORPORATIVAS}

Como se ha indicado en la metodología, el trabajo de Aichner y Jacob (2015) evaluó las pla- taformas sociales y blogs usados por las empresas. Dentro de su estudio realizaron una ponderación de cada tipología de plataforma social respecto la afectación a la función corporativa de las organizaciones. De esta forma, categorizando el grado de vinculación, se ha aplicado una ponderación ${ }^{8}$ de las funciones corporativas relacionadas con el uso de cada una de las plataformas sociales usadas por las empresas del IBEX 35.

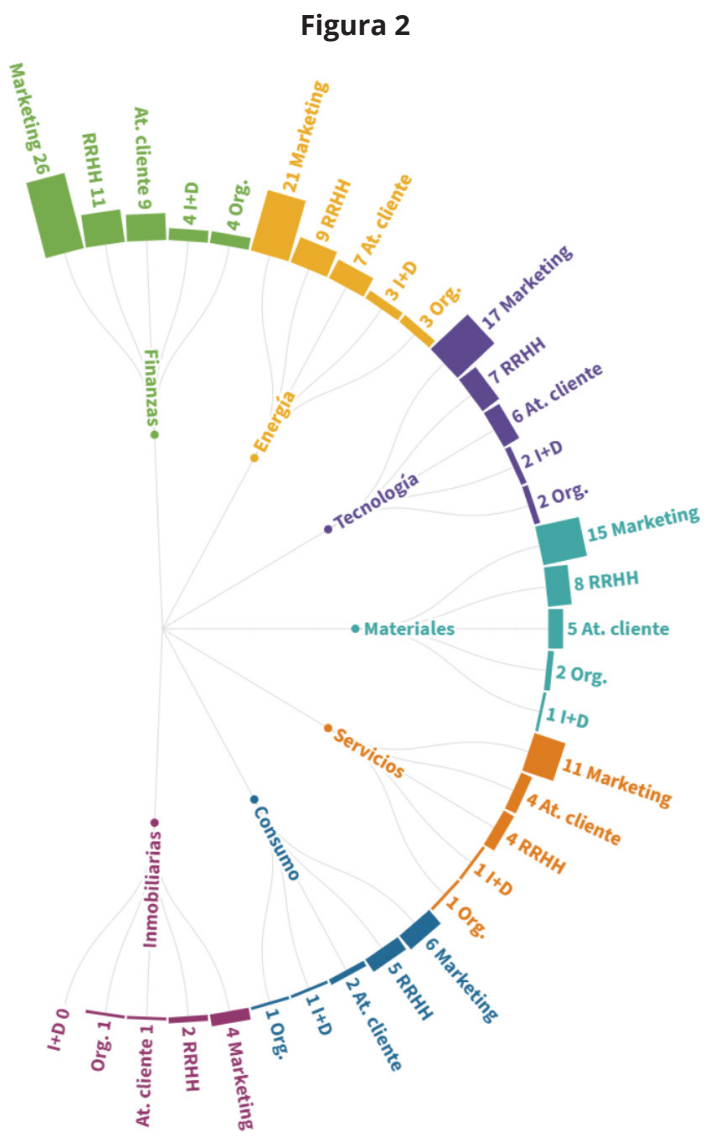

Nota: Gráfica radial distribuida por sectores con ponderación de las funciones corporativas en relación con el uso de redes sociales por parte de las organizaciones del IBEX 35.
8 Como se comentó en el apartado Metodología el ratio de importancia en cada función corporativa se aplicó siguiendo la propuesta de Alchnar y Jacob (2015) de la siguiente manera: 0 (ninguna importancia), 0,25 (baja), 0,5 (media), 0,75 (alta) y 1 (muy alta). 
Como indica claramente la figura 2 la función de Marketing aparece como la más importante en relación a la tipología de redes sociales usadas por las empresas del IBEX 35. En segundo y tercer lugar estarían las actividades de recursos humanos y gestión del talento, y la atención al cliente usando redes sociales.

\subsection{LA CAPITALIZACIÓN COMO CONDICIONANTE EN EL USO DE REDES SOCIALES CORPORATIVAS}

Aunque existe una nutrida bibliografía sobre la relación entre tamaño de las organizaciones y uso de redes sociales, especialmente en el caso de pequeñas y medianas empresas (Ahmad et al., 2018; Behan, 2014; Karimi \& Naghibi, 2015; Meske \& Stieglitz, 2013; Roy et al., 2014; Srinivasan et al., 2016) no existen trabajos que correlacionen directamente la dimensión económica con la mayor o menor utilización de redes sociales. Para dar una respuesta parcial a esta disyuntiva esta investigación ha extraído los datos de capitalización bursátil y se ha combinado con la aceptación o no aceptación de cada una de las redes sociales que forman parte del ecosistema digital de estas organizaciones.

Tomando como muestra las empresas que componen el IBEX 35, la introducción del componente económico subvierte parcialmente los resultados obtenidos en los que se ponderan las diferentes empresas con el mismo peso. Como puede apreciarse en la siguiente figura, si se cuantifica la capitalización bursátil de cada una de las compañías integrantes de la muestra se produce un cambio relevante en la hegemonía de las plataformas sociales más importantes usadas en el IBEX 35.
Figura 3

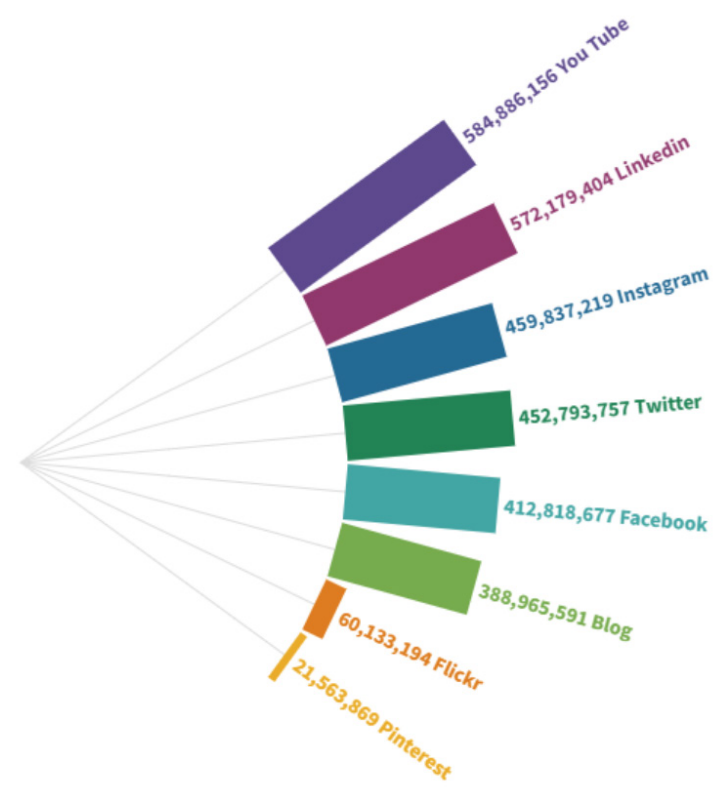

Nota: Gráfica radial organizada cuantitativamente según capitalización acumulada en miles de euros de las redes sociales usadas por parte de las organizaciones del IBEX 35.

Al introducir una ponderación por capitalización de las compañías se puede observar que YouTube destaca en solitario como red social con mayor uso corporativo seguido a corta distancia por Linkedln, ya en un rango inferior figuran Instagram, Twitter, Facebook, Blogs y finalmente con presencia residual Flickr y Pinterest.

La cuestión más relevante que emerge en la inclusión de la variable de capitalización sugiere la apuesta creciente de las compañías de mayor tamaño por el uso de Instagram, ya que la ponderación por capitalización coloca esta plataforma en tercer lugar, pese a haber un número sensiblemente inferior de empresas que apuestan por este canal (figura 1), existiendo, por tanto, una correlación entre el tamaño de las empresas y la aceptación del uso de Instagram como vía de comunicación. 
Para poder evaluar con mayor profundidad estos resultados se aplicó una ordenación por cuartiles ${ }^{9}$ de la capitalización bursátil y de otras variables que fueran medibles cuantitativamente. La agrupación en cuartiles permite la categorización de un subgrupo de datos en relación a la población total de datos. De esta forma, como indican Díez y Moulines (2008), un concepto científico de tipo métrico (cuantitativo) permite obtener un concepto de tipo comparativo (cualitativo) y este a su vez uno clasificatorio (cualitativo).

Las variables que se cuantificaron y agruparon en cuartiles además de la ya mencionada dimensión financiera fueron el número de visitas a la página web oficial ${ }^{10}$, el número de seguidores en Twitter, el número de «me gusta» en Facebook, el número de seguidores en Linkedln y el número de seguidores en Instagram. Estos datos se pusieron en diálogo dentro de una misma estructura de datos y se visualizaron gracias a una gráfica aluvial11.

Figura 4

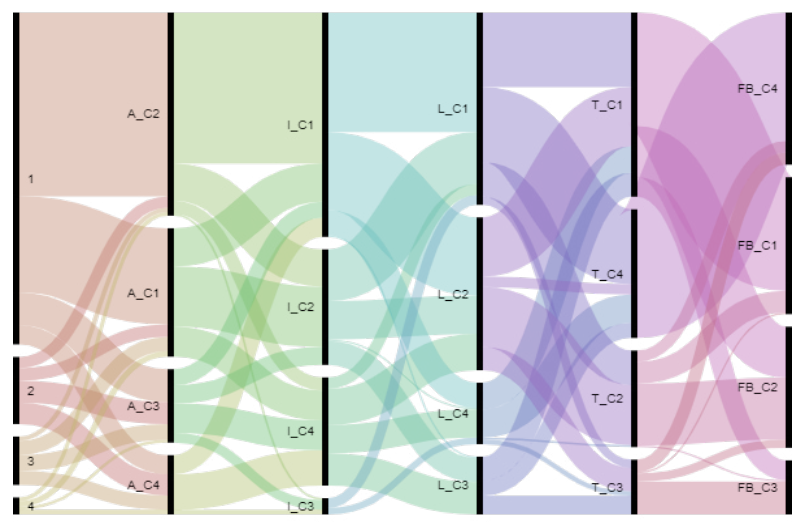

Nota: Gráfica aluvial ordenada según capitalización acumulada de las empresas que forman parte de cada cuartil. \#: Cuartil de capitalización, A_C\#: cuartil Alexa, I_C\#: cuartil Instagram, L_C\#: cuartil Linkedin, FB_C\#: cuartil Facebook.

9 División de un conjunto de datos ordenados numéricamente en cuatro partes iguales.

10 Medido según el ranking atribuido por el portal de tráfico web Alexa.

11 Diagrama de flujo que representa cambios en una estructura de la red al pasar de una fase a otra.
Como puede observarse, el primer nivel corresponde con la ordenación por capitalización en el que la primera agrupación, pese a estar distribuida uniformemente, absorbe el peso de la capitalización bursátil de gran parte de la cotización del IBEX 35. Las siguientes ordenaciones basadas en variables cuantitativas mantienen sin grandes cambios una jerarquía hegemónica en que las empresas con mayor capitalización tienden a ser las que tienen un mayor número de visitas en su web oficial (ordenación del segundo nivel según ranking Alexa), seguidores en Instagram (tercer nivel) y seguidores en Linkedln (cuarto nivel). Ahora bien, si se pone el foco en el quinto nivel (seguidores en Twitter) puede argumentarse un cambio de tendencia pues existe una correlación negativa entre número de seguidores en Twitter y capitalización. El grupo de empresas con un menor número de seguidores en Twitter (o directamente que no usan esta plataforma) se encuentra en el segundo nivel de importancia según su capitalización bursátil.

Esta misma dinámica, pero todavía más significativa, se produce en el sexto nivel organizado por el número de usuarios de Facebook. Las empresas que tienen un menor número de seguidores o que no aceptan esta plataforma dentro de su política de comunicación poseen una capitalización superior que todas las demás agrupaciones de empresas, incluidas las que tienen un mayor número de usuarios. Este fenómeno contraviene la naturaleza común en el resto de parámetros según la cual las empresas con mayor masa financiera obtienen también mejores ratios en visitas web y seguidores en redes sociales.

A su vez la distribución de Facebook y en menor medida de Twitter resulta más reveladora cuando la capitalización acumulada entre estas dos plataformas e Instagram apenas está descompensada tal como puede apreciarse en la 
figura 3. Es decir, aunque no existan grandes diferencias en la capitalización acumulada de estos tres canales, la ordenación por cuartiles señala una evidente correlación positiva entre seguidores y capitalización en el caso de Instagram y una correlación negativa en el caso de Facebook (y en menor medida en Twitter).

La conclusión provisional que puede tomarse de este fenómeno indica a pensar que, de nuevo, existe una política de las grandes empresas (y dentro de estas las económicamente más importantes) a apostar por canales con una mayor dimensión unidireccional en detrimento de la perspectiva conversacional. Este indicio se sustenta en que Facebook es el canal con una mayor interacción si esta es medida como acceso de los usuarios activos durante más de una vez al día12 (50-43\% de usuarios). Twitter estaría en segundo lugar en cuanto a la interacción respecto a las plataformas incluidas en la organización por cuartiles con un $27 \%$ de acceso, seguida de Instagram con un $24 \%$ y Linkedin con un 13\%. Por tanto, se produce una correlación inversa entre nivel de interacciones en la plataforma (dimensión conversacional) y aceptación por las empresas con mayor capitalización (dimensión económica).

Esta argumentación es debida a que Linkedln e Instagram mantienen una estructura en que el primer cuartil por usuarios es también la mayor agrupación por capitalización, aunque el tercer y cuarto cuartil hayan intercambiado posiciones. En el caso de Twitter el primer cuartil por usuarios sigue estando correlacionado con una mayor capitalización, si bien el cuartil con menos usuarios obtiene un segundo orden de importancia por capitalización. Finalmente, en Facebook el cuartil que contiene las cuentas con menor número de usuarios o que direc-

12 Recuperado de https://bit.ly/2Kupbfv el 20 de febrero de 2020. tamente no reconoce esta plataforma tiene la mayor capitalización acumulada tomando como muestra las empresas del IBEX 35 que son a su vez las que tienen un mayor valor patrimonial en España.

\section{DISCUSIÓN Y CONCLUSIONES}

Esta investigación se ha propuesto una observación del ecosistema de redes sociales de las empresas del IBEX 35, tanto de aquellas promocionadas en su web oficial como de aquellas únicamente reconocidas en mayor o menor grado (redes sociales o blogs).

Las conclusiones pueden exponerse mediante varios puntos clave. Por una parte, tal como han reseñado estudios anteriores enfocados a las empresas de este índice bursátil (Aced Toledano, 2017; Durántez-Stolle, 2017) existe una amplia aceptación de las redes sociales dentro de la política de comunicación de estas compañías. Incluso es reconocible un ligero aumento en los últimos años si se valora la evolución de la tecnología y el cambio de marco metodológico.

Por otra parte, el no reconocimiento explícito de los blogs corporativos es un fenómeno generalizado y representa una política que coincide con el mismo formato de los sitios web en los que se busca una promoción no evidente de la imagen de la compañía. Los blogs, según la muestra analizada, son usados como vías paralelas a la actividad de comunicación previa a la aparición del escenario 2.0, pero evitando una visibilidad evidente de las compañías promotoras. Esta característica puede relacionarse con su perspectiva eminentemente unidireccional en comparación con las redes sociales.

La función del marketing es preeminente según los datos de esta investigación si se convierte el 
uso de plataformas sociales en funciones corporativas según la propuesta de Aichner y Jacob (2015). Esta cuestión reafirma tangencialmente la lógica según la cual las grandes empresas como las que pertenecen al IBEX 35 mantienen una visión de traslación de información por encima de un enfoque conversacional. Esta conclusión se encuentra fundamentada en que la conversión de la propuesta de Aichner y Jacob (2015) a coeficientes deja la función de Atención al cliente en un nivel claramente inferior ${ }^{13}$.

Finalmente, la dimensión económica valorada en esta investigación confirma empíricamente la conclusión de los estudios de Aced Toledano (2017) y Durántez-Stolle (2017). En los trabajos de estos dos autores se concluyó que las grandes empresas hacen uso de las redes sociales desde una perspectiva más informativa que no como espacio para la generación de interacciones y diálogo. El presente trabajo ha coincidido en semejante hallazgo ya que, si bien la muestra analizada está basada sólo en empresas de gran tamaño, dentro de este mismo abanico puede observarse que Facebook, como red social que genera más actividad diaria, es la más importante en número de seguidores para las empresas que tienen una capitalización menor. Por el contrario, dos plataformas con una interacción claramente inferior, Linkedln e Instagram, se encuentran en la cúspide por usuarios para las compañías con mayor capitalización.

De esta forma este estudio aporta un indicio significativo basado en datos constatables y que corrobora conclusiones previas obtenidas mediante otras metodologías. Ahora bien, conviene reseñar que esta investigación se ha sustentado en una muestra limitada a las compañías de mayor capitalización en España en un momento determinado. De la misma forma que esta cuestión evita la formulación de generalizaciones, abre la puerta a futuros trabajos que confronten la categoría de las empresas analizadas con compañías de lugares o de características diferentes como por ejemplo empresas emergentes.

13 Siempre en tercer lugar tras las funciones de Marketing y Recursos Humanos exceptuando en el sector de empresas del IBEX catalogadas como Servicios en que queda sólo por debajo de Marketing. 


\section{REFERENCIAS}

Aced Toledano, C. (2017). How Companies Are Seizing the Dialogic Opportunities Provided by Social Media to Communicate with their External Audiences (Tesis doctoral, Universitat Oberta de Catalunya).

Ahmad, S. Z., Ahmad, N., \& Bakar, A. R. A. (2018). Reflections of entrepreneurs of small and medium-sized enterprises concerning the adoption of social media and its impact on performance outcomes: Evidence from the UAE. Telematics and Informatics, 35(1), 6-17 https://doi.org/10.1016/j. tele.2017.09.006

Aichner, T., \& Jacob, F. (2015). Measuring the degree of corporate social media use. International Journal of Market Research, 57(2), 257-276. https:// doi.org/10.2501/ijmr-2015-018

Alalwan, A. A. (2018). Investigating the impact of social media advertising features on customer purchase intention. International Journal of Information Management, 42, 65-77. https://doi.org/10.1016/j.ijinfomgt.2018.06.001

Allagui, I., \& Breslow, H. (2016). Social media for public relations: Lessons from four effective cases. Public Relations Review, 42(1), 20-30. https:// doi.org/10.1016/j.pubrev.2015.12.001

Alonso, E. L., \& López, B. M. (2019). La gestión de los medios sociales en la Administración local: análisis de los municipios rurales españoles. Vivat Academia, 148, 77-99. https://doi.org/10.15178/va.2019.148.77-99

Barris, K. (2019). Using stakeholder theory to understanding B2B social media usage in South Africa (Tesis doctoral, University of Pretoria).

Bartov, E., Faurel, L., \& Mohanram, P. S. (2018). Can Twitter help predict firm-level earnings and stock returns? The Accounting Review, 93(3), 2557. https://doi.org/10.2139/ssrn.2782236

Behan, M. K. (2013). Efficacy of social media in participant perception of brand in small to medium size enterprises (Tesis doctoral Capella University).

Cade, N. L. (2018). Corporate social media: How two-way disclosure channels influence investors. Accounting, Organizations and Society, 68, 63-79. https://doi.org/10.2139/ssrn.2619249

Castelló Martínez, A. (2013). Estrategias empresariales en la Web 2.0. Las redes sociales Online. Alicante, España: Editorial Club Universitario.

Chen, H., De, P., Hu, Y. J., \& Hwang, B. H. (2014). Wisdom of crowds: The value of stock opinions transmitted through social media. The Review of Financial Studies, 27(5), 1367-1403. https://doi.org/10.1093/rfs/hhu001 
Costa, J. (2016). Para comprender el Dircom. Intersecciones en comunicación, 5(5), 161-178

Deuze, M. (2008). Corporate appropriation of participatory culture, en Carpentier, N., Livingstone, S. (eds.), Participation and Media Production: Critical Reflections on Content Creation. Newcastle upon Tyne: Cambridge Scholars Publishers.

Díez, J. A., \& Moulines, C. U. (2008). Fundamentos de filosofía de la ciencia (No. 165). Barcelona, España: Ariel.

Durántez-Stolle, P. (2017). Redes sociales virtuales en la comunicación corporativa del IBEX 35: usos, desarrollo e importancia según sus responsables. Revista Mediterránea de Comunicación, 8(1), 137-154. https://doi. org/10.14198/medcom2017.8.1.10

Enke, N., \& Borchers, N. S. (2019). Social media influencers in strategic communication: A conceptual framework for strategic social media influencer communication. International Journal of Strategic Communication, 13(4), 261-277. https://doi.org/10.1080/1553118X.2019.1620234

Flores Vizcarra, H. A. (2019). Análisis de la Influencia de Medios Sociales en la Reputación Corporativa de las Empresas Operadoras Móviles en el Perú-2019.

Hamid, S. (2020). The Routledge Handbook of Strategic Communication. Pakistan Review of Social Sciences (PRSS), 1(1), 4-5.

Karimi, S., \& Naghibi, H. S. (2015). Social media marketing (SMM) strategies for small to medium enterprises (SMEs). International Journal of Information, Business and Management, 7(4), 86.

Kick, M. (2015). Selected essays on corporate reputation and social media. Wiesbaden, Alemania: Springer Books.

Korzynski, P., Mazurek, G., \& Haenlein, M. (2020). Leveraging employees as spokespeople in your HR strategy: How company-related employee posts on social media can help firms to attract new talent. European Management Journal, 38(1), 204-212. https://doi.org/10.1016/j. emj.2019.08.003

Klewes, J., Popp, D., \& Rost-Hein, M. (2017). Out-thinking organizational communications. The Impact of Digital Transformation. Berlín, Alemania: Springer International Publishing.

Lei, L. G., Li, Y., \& Luo, Y. (2019). Production and dissemination of corporate information in social media: A review. Journal of Accounting Literature, 42, 29-43. https://doi.org/10.2139/ssrn.3330875

Macnamara, J. (2010). Public relations and the social: How practitioners are using, or abusing, social media. Asia Pacific Public Relations Journal, 11, 21-39. 
Meske, C., \& Stieglitz, S. (2013). Adoption and use of social media in small and medium-sized enterprises. En working conference on practice-driven research on enterprise transformation (pp. 61-75). Berlin, Heidelberg.

Miller, G. S., \& Skinner, D. J. (2015). The evolving disclosure landscape: How changes in technology, the media, and capital markets are affecting disclosure. Journal of Accounting Research, 53(2), 221-239. https://doi. org/10.2139/ssrn.2560855

Okazaki, S., Plangger, K., West, D., \& Menéndez, H. D. (2020). Exploring digital corporate social responsibility communications on Twitter. Journal of Business Research, 117, 675-682. https://doi.org/10.1016/j.jbusres.2019.09.006

Paliwoda-Matiolanska, A., Smolak-Lozano, E., \& Nakayama, A. (2020). Corporate image or social engagement: Twitter discourse on corporate social responsibility (CSR) in public relations strategies in the energy sector. Profesional de la Información, 29(3). https://doi.org/10.3145/ epi.2020.may.33

Poppel, H. (2020). An Examination of Enterprise Social Media and its Impact on Employees in a Global Corporate Organization (Tesis doctoral, Concordia University St. Paul).

Postman, J. (2009). SocialCorp: Social media goes corporate. Berkeley: New Riders.

Roy, A., Maxwell, L., \& Carson, M. (2014). How is social media being used by small and medium-sized enterprises? Journal of business and behavioral sciences, 26(2), 127.

Srinivasan, R., Bajaj, R., \& Bhanot, S. (2016). Impact of social media marketing strategies used by micro small and medium enterprises (MSMEs) on Customer acquisition and retention. IOSR Journal of Business and Management, 18(1), 91-101. https://doi.org/10.9790/487X-181391101

Tom, A. A., Vijayakrishnan, G., \& Thangjam, R. (2018). Effect of Twitter tweets on the short term stock prices after Donald Trump's presidency. International Journal of Research and Analytical Reviews 5, (4), 46-55.

Torres Ponjuán, D. (2010). La visualización de la información en el entorno de la Ciencia de la Información. (Tesis doctoral, Universidad de Granada).

Túñez López, M., \& Altamirano, V. (2015). La simetría interactiva de la comunicación organizacional. Revista DIRCOM, (108), 14-17.

Uttrup, E. B. (2017). A digital communication plan for Quiksilver, Portugal (Tesis doctoral, Instituto Universitário de Lisboa).

Visser, M., \& Sikkenga, B. (2017). Social media management vanuit commercieel perspectief. Groningen: Noordhoff Uitgevers. 
Yang, T., \& Yang, Y. (2017). Predict Effect of Trump's Tweets on Stock Price Milestone (Tesis de pregrado, Stanford University).

Wang, Y., \& Yang, Y. (2020). Dialogic communication on social media: How organizations use Twitter to build dialogic relationships with their publics. Computers in Human Behavior, 104, 106183. https://doi. org/10.1016/j.chb.2019.106183

ANEXO 1: Datos obtenidos en la investigación

Tabla 1

\begin{tabular}{|c|c|c|c|c|c|}
\hline Nombre & $\begin{array}{c}\text { Ranking } \\
\text { Alexa }\end{array}$ & $\begin{array}{c}\text { Seguidores } \\
\text { Linkedin }\end{array}$ & $\begin{array}{l}\text { Seguidores } \\
\text { Instagram }\end{array}$ & $\begin{array}{l}\text { "me gusta" } \\
\text { en Facebook }\end{array}$ & Capitalización \\
\hline Inditex & 87.825 & 917.839 & 68.003 & 0 & 96.709 .712 \\
\hline Iberdrola & 57.390 & 219.083 & 33.674 & 38.738 & 60.159 .753 \\
\hline Banco Santander & 3.015 & 783.193 & 20.416 & 233.000 & 59.442 .996 \\
\hline Amadeus & 2.447 & 222.909 & 0 & 70.908 & 33.431 .929 \\
\hline Telefónica & 49.494 & 8.025 & 25.890 & 843.000 & 32.539 .089 \\
\hline BBVA & 3.939 & 467.363 & 165.687 & 5.500 .000 & 31.325 .731 \\
\hline AENA & 37.007 & 38.743 & 11.122 & 18.829 & 25.462 .500 \\
\hline ENDESA & 621.249 & 151.279 & 47.926 & 25.940 & 24.590 .000 \\
\hline Naturgy & 64.454 & 89.516 & 8.732 & 74.138 & 22.546 .238 \\
\hline Ferrovial & 266.079 & 253.375 & 6.606 & 79.248 & 21.563 .869 \\
\hline REPSOL & 166.515 & 475.456 & 34.428 & 171.322 & 20.772 .586 \\
\hline Cellnex & 2.849 .154 & 19.070 & 0 & 0 & 16.861 .889 \\
\hline Caixabank & 1.561 & 97.474 & 97.262 & 182.000 & 16.149 .883 \\
\hline IAG & 477.690 & 0 & 0 & 0 & 15.015 .942 \\
\hline Arcelor & 65.558 & 401.112 & 0 & 0 & 14.537 .601 \\
\hline Grifols & 392.050 & 114.733 & 0 & 0 & 13.457 .179 \\
\hline$A C S$ & 808.686 & 0 & 0 & 0 & 10.604 .197 \\
\hline Siemens Gamesa & 337.394 & 295.470 & 5.957 & 22.156 & 10.595 .185 \\
\hline Red Eléctrica & 570.290 & 32.531 & 0 & 3.605 & 9.728 .618 \\
\hline Mapfre & 35.130 & 296.791 & 20.255 & 1.900 .000 & 7.424 .803 \\
\hline ENAGAS & 1.078 .202 & 49.114 & 0 & 1.278 & 6.298 .241 \\
\hline Colonial & 8.793 .564 & 6.939 & 1.067 & 0 & 5.980 .511 \\
\hline Merlin & 8.793 .564 & 4.134 & 0 & 0 & 5.942 .600 \\
\hline Acciona & 116.808 & 477.674 & 134.102 & 450.000 & 5.556 .979 \\
\hline Bankinter & 9.012 & 49.141 & 10.085 & 105.000 & 5.465 .106 \\
\hline Banc Sabadell & 3.569 & 105.380 & 6.786 & 66.141 & 5.459 .281 \\
\hline Bankia & 4.823 & 32.647 & 0 & 36.502 & 5.278 .043 \\
\hline $\mathrm{CIE}$ & 1.187 .248 & 16.106 & 0 & 0 & 2.714 .160 \\
\hline Acerinox & 598.471 & 6.208 & 0 & 0 & 2.595 .079 \\
\hline Más Móvil & 81.213 & 3.508 & 4.419 & 133.000 & 2.522 .334 \\
\hline Viscofan & 151.559 & 7.262 & 0 & 0 & 2.309 .190 \\
\hline Indra & 216.322 & 497.455 & 4.049 & 21.602 & 1.950 .265 \\
\hline Melia & 27.472 & 34.257 & 110.003 & 873.000 & 1.873 .204 \\
\hline Mediaset & 174.876 & 0 & 20.663 & 157.589 & 1.747 .194 \\
\hline Ence & 4.304 .427 & 21.514 & 0 & 2775 & 934.850 \\
\hline
\end{tabular}

Nota: datos cuantitativos de las redes sociales principales en las empresas del IBEX 35 agrupados por capitalización de cada compañía. Ranking Alexa, número de seguidores en Linkedln, número de

seguidores en Instagram, número de "me gusta" en Facebook y capitalización en bolsa (en millones de euros). 